\title{
Correction to: Effectiveness of working memory training among children with dyscalculia: evidence for transfer effects on mathematical achievement-a pilot study
}

\author{
Smail Layes ${ }^{1,2} \cdot$ Robert Lalonde $^{2,3} \cdot$ Yamina Bouakkaz $^{1} \cdot$ Mohamed Rebai $^{2}$ \\ Published online: 8 February 2019 \\ ( ) Marta Olivetti Belardinelli and Springer-Verlag GmbH Germany, part of Springer Nature 2019
}

\section{Correction to: Cognitive Processing (2018) 19:375-385 https://doi.org/10.1007/s10339-017-0853-2}

The following errors require correction in the article. The authors apologize for these errors.

1. In the abstract, the term "dyslexia" should be replaced by the term "dyscalculia". Furthermore, the mean age of the experimental group was not " 129.74 months" but "115.29 months", whereas the mean age of the control group was not "126.9 months" but "116.07 months".

2. In the section "Participants" the mean ages of the experimental group and of the control group should accordingly be replaced by " 115.29 months ( $\mathrm{SD}=3.60$ months)" and "116.07 months ( $\mathrm{SD}=1.07$ months)", respectively.

3. In the section "Training Effects", $t$ (2.05) should be replaced by $t(26)$, and $t(15)$ should be replaced by $t$ (13).

The original article can be found online at https://doi.org/10.1007/ s10339-017-0853-2.

Smail Layes

smail.layes@gmail.com

Robert Lalonde

robert.lalonde@univ-rouen.fr

Yamina Bouakkaz

yaminabouakaz70@gmail.com

Mohamed Rebai

mohamed.rebai@univ-rouen.fr

1 School of Psychology, University of El-Oued, El-Oued, Algeria

2 CRFDP, Normandie Université, Rouen, France

3 UFR des Sciences de l'Homme et de la société, Place Emile Blondel, Bât Freinet, 76821 Mont Saint Aignan Cedex, France
4. In Table 2, the titles "Control group" and "Experimental group" should be swapped, and the title "Difference $(=2.05)$ (ns)" should be replaced by "Difference, $t$ (26)". In the row "Age", " $M=116.00, \mathrm{SD}=.00$ " should be replaced by " $M=116.07, \mathrm{SD}=1.07$ ", and " $t=-.74$ " should be replaced by " $t=.78$ ". In the row "Mental calculation", " $M=9.43$, SD 1.15 " should be replaced by " $M=9.57, \mathrm{SD}=1.08 "$. The correct table is reproduced below.

5. In Table 3, the titles "Control group" and "Experimental group" should be swapped. In the row "Dictation of number", Cohen's d " 0.25 " should be replaced by "2.50". In the row "Total math", " $\mathrm{M}=38.36, \mathrm{SD}=1.59$ " should be replaced by " $\mathrm{M}=38.57, \mathrm{SD}=1.82$ ". The correct table is reproduced below.

Table 2 Differences between the experimental and control groups in pre-intervention measures

\begin{tabular}{|c|c|c|c|c|c|}
\hline \multirow[t]{2}{*}{ Measures } & \multicolumn{2}{|c|}{$\begin{array}{l}\text { Control } \\
\text { group }\end{array}$} & \multicolumn{2}{|c|}{$\begin{array}{l}\text { Experimental } \\
\text { group }\end{array}$} & \multirow[t]{2}{*}{$\begin{array}{l}\text { Difference } \\
t(26)(\mathrm{ns})\end{array}$} \\
\hline & $M$ & SD & $M$ & SD & \\
\hline Age & 116.07 & 1.07 & 115.29 & 3.60 & .78 \\
\hline Raven & 28.57 & .64 & 28.71 & 3.70 & .14 \\
\hline Counting backward & 2.50 & .51 & 2.36 & 1.00 & .47 \\
\hline Dictation of number & 8.71 & .46 & 9.00 & 1.30 & .77 \\
\hline Mental calculation & 9.57 & 1.08 & 9.57 & 2.06 & .22 \\
\hline Reading numbers & 9.43 & .51 & 9.29 & 3.19 & .16 \\
\hline Writ. number comparison & 7.36 & .49 & 7.07 & 1.20 & .81 \\
\hline Total Math & 37.57 & 1.39 & 37.29 & 5.26 & .19 \\
\hline Forward digit span & 6.14 & .86 & 5.86 & .77 & .92 \\
\hline Backward digit span & 4.71 & .46 & 4.86 & .66 & 65 \\
\hline Total WM & 10.86 & .86 & 10.71 & 1.13 & .37 \\
\hline
\end{tabular}


Table 3 Differences between the experimental and control groups in post-intervention measures

\begin{tabular}{|c|c|c|c|c|c|c|}
\hline \multirow[t]{2}{*}{ Measures } & \multicolumn{2}{|c|}{ Control group } & \multicolumn{2}{|c|}{$\begin{array}{l}\text { Experimental } \\
\text { group }\end{array}$} & \multirow[t]{2}{*}{ Difference $(t=2.05)$} & \multirow[t]{2}{*}{ Cohen's $d$} \\
\hline & $M$ & (SD) & $M$ & $(\mathrm{SD})$ & & \\
\hline Raven & 29.00 & .68 & 30.64 & 2.40 & $2.24 *$ & 0.85 \\
\hline Counting backward & 2.57 & .51 & 3.93 & .26 & $8.77 * * *$ & 3.35 \\
\hline Dictation of numbers & 8.86 & .66 & 12.86 & 2.17 & $6.57 * * *$ & 2.50 \\
\hline Mental calculation & 9.64 & 1.21 & 13.29 & 1.49 & $7.08 * * *$ & 2.68 \\
\hline Reading numbers & 9.71 & .61 & 12.71 & .99 & $9.61 * * *$ & 3.64 \\
\hline Writ. number comparison & 7.57 & .51 & 8.71 & .46 & $6.15^{* * *}$ & 2.34 \\
\hline Total Math & 38.57 & 1.82 & 51.50 & 4.20 & $10.93 * * *$ & 4.38 \\
\hline Forward digit span & 6.29 & .91 & 9.86 & 1.29 & $8.44 * * *$ & 3.19 \\
\hline Backward digit span & 4.93 & .26 & 9.29 & 1.63 & $9.82 * * *$ & 3.73 \\
\hline Total WM & 11.21 & .89 & 19.14 & 2.59 & $10.80 * * *$ & 4.09 \\
\hline
\end{tabular}

$* p<.05 ; * * p<.01 ; * * * p<.001$

\begin{tabular}{|c|c|c|c|c|c|c|}
\hline \multirow[t]{2}{*}{ Measures } & \multicolumn{3}{|c|}{ Control group } & \multicolumn{3}{|c|}{ Experimental group } \\
\hline & Difference & $t$ value & Cohen's $d$ & Difference & $t$ value & Cohen's $d$ \\
\hline Raven & .43 & $2.48^{*}$ & 0.65 & 1.92 & $3.22 * * *$ & 0.61 \\
\hline Counting backward & .07 & .56 & & 1.57 & $5.39 * * *$ & 2.14 \\
\hline Dictation of number & .14 & .69 & & 3.85 & $6.32 * * *$ & 2.15 \\
\hline Mental calculation & .21 & .56 & & 3.71 & $5.71 * * *$ & 2.06 \\
\hline Reading numbers & .28 & $2.28 *$ & 0.49 & 3.42 & $3.53 * * *$ & 1.44 \\
\hline Writ. number comp & .21 & 1.38 & & 1.64 & $5.34 * * *$ & 1.80 \\
\hline Total Math & .79 & 2.13 & & 14.21 & $8.69 * * *$ & 2.98 \\
\hline Forward digit span & .14 & .80 & & 4.00 & $11.01 * * *$ & 3.76 \\
\hline Backward digit span & .21 & 1.88 & & 4.42 & $11.01 * * *$ & 3.56 \\
\hline Total WM & .35 & 1.79 & & 8.42 & $12.44 * * *$ & 3.56 \\
\hline
\end{tabular}

$* p<.05 ; * * p<.01 ; * * * p<.001$
Table 4 Differences between pre- and post-intervention measures for the experimental and control groups
Publisher's Note Springer Nature remains neutral with regard to jurisdictional claims in published maps and institutional affiliations.
6. In Table 4, all negative values should be positive. In the row "Total math", "- 1.00 " should be replaced by ".79". The correct table is reproduced below. 